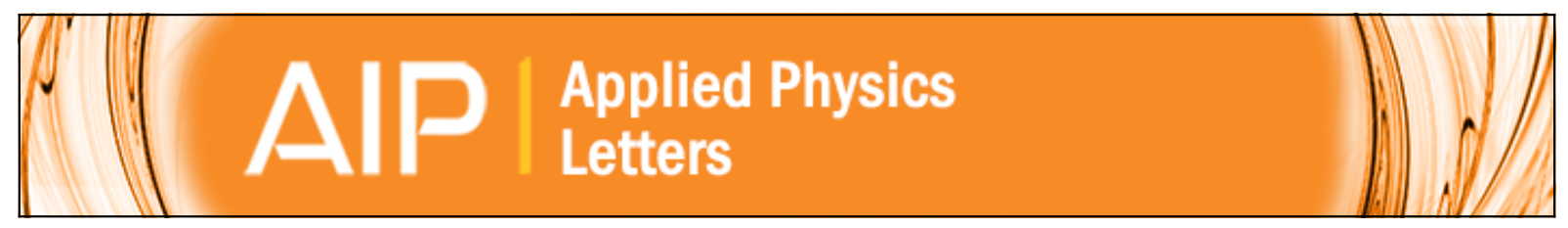

\title{
A study of the pair distribution function of self-organized Ge quantum dots
}

Marco Bernardi, Anna Sgarlata, Massimo Fanfoni, Adalberto Balzarotti, and Nunzio Motta

Citation: Applied Physics Letters 93, 031917 (2008); doi: 10.1063/1.2965122

View online: http://dx.doi.org/10.1063/1.2965122

View Table of Contents: http://scitation.aip.org/content/aip/journal/apl/93/3?ver=pdfcov

Published by the AIP Publishing

\section{Articles you may be interested in}

Deterministic control of plasma-assembled self-organized $\mathrm{Ge} / \mathrm{Si}$ quantum dot arrays

J. Appl. Phys. 101, 094309 (2007); 10.1063/1.2727448

Self-assembly of Ge quantum dots on Si(100)- $2 \times 1$ by pulsed laser deposition

Appl. Phys. Lett. 86, 243104 (2005); 10.1063/1.1949285

Optical and acoustic phonon modes in self-organized Ge quantum dot superlattices

Appl. Phys. Lett. 76, 586 (2000); 10.1063/1.125825

Controlled arrangement of self-organized $\mathrm{Ge}$ islands on patterned $\mathrm{Si}(001)$ substrates

Appl. Phys. Lett. 75, 2752 (1999); 10.1063/1.125138

Growth and study of self-organized Ge quantum wires on $\mathrm{Si}(111)$ substrates

Appl. Phys. Lett. 74, 2471 (1999); 10.1063/1.123884

\section{AlP $\mid$ APL Photonics}

APL Photonics is pleased to announce Benjamin Eggleton as its Editor-in-Chief

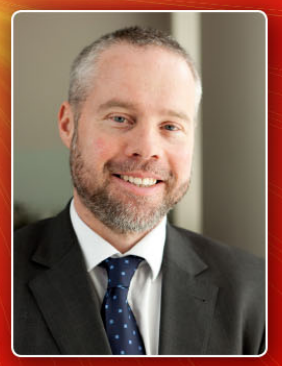




\title{
A study of the pair distribution function of self-organized Ge quantum dots
}

\author{
Marco Bernardi, ${ }^{1, a)}$ Anna Sgarlata, ${ }^{1}$ Massimo Fanfoni, ${ }^{1}$ Adalberto Balzarotti, ${ }^{1}$ and \\ Nunzio Motta ${ }^{2}$ \\ ${ }^{1}$ Dipartimento di Fisica, Università di Roma Tor Vergata, Via della Ricerca Scientifica 1, 00133 Roma, Italy \\ ${ }^{2}$ School of Engineering Systems, Queensland University of Technology, GPO BOX 2434, \\ Brisbane 4001, Australia
}

(Received 21 May 2008; accepted 7 July 2008; published online 25 July 2008)

\begin{abstract}
We explore the use of the pair distribution function to study the self-organization process of Ge quantum dots on both nanopatterned and nonpatterned oxidized $\mathrm{Si}(001)$ surfaces. Dots formation and ordering upon annealing of a Ge thin film are analyzed. The method we use is not limited to this case study. We show how it can be applied to determine short and long range self-ordering of nanostructures. We support our results by applying a software routine to simulate patterns of dots to finally spot the relevant physical aspects of Ge islands self-assembly. (C) 2008 American Institute of Physics. [DOI: 10.1063/1.2965122]
\end{abstract}

Self-organization of metal nanoparticles, ceramic nanoislands, and semiconductor quantum dots (QDs) at surfaces is a phenomenon of relevance in materials science. ${ }^{1-3}$ The attention received by these systems of nanostructures stems both from the inspiring findings related to the selfassembly process itself and from their tremendous technological potential. ${ }^{4,5}$ While the display of appealing physical and chemical properties originally triggered the investigation of QDs, it is now equally important for the development of several QDs-based nanoelectronic, optoelectronic, and nanolithography applications to acquire the capability of fast, parallel fabrication of high-density arrays of laterally ordered nanoislands. ${ }^{6,7}$ This architecture is likely to result from bottom-up, self-assembly processes in which nanodots ordering is driven from internal fluxes and forces, like the stress field occurring at $\mathrm{Si}-\mathrm{Ge}$ interface during $\mathrm{Ge} / \mathrm{Si}$ heteroepitaxy, perhaps coupled to some form of nanopatterning. ${ }^{8-10}$ Therefore, the acquisition of an ultimate control and understanding of in-plane interactions of QDs at surfaces is an urgent need for nanotechnology, and the detection of any degree of self-ordering among nanostructures, which may well elude visual inspection, is of great help in this undertaking.

Within this framework, considerable efforts have been devoted to characterize and simulate QDs self-organization using a variety of physical and theoretical means. ${ }^{11,12} \mathrm{Al}-$ though a recently reported numerical approach was able to detect the presence of short-range order (SRO) among nanostructures, ${ }^{13,14}$ a single methodology to assess both SRO and long-range order (LRO) is still lacking, thus utterly impeding the study of many systems of technological interest and precluding the evaluation of long range interactions. ${ }^{15}$ Here, we pursue an efficient and direct approach to estimate the pair distribution function (PDF) of an assembly of QDs on a surface with any degree of ordering and we examine both their SRO and their LRO. The PDF is a basic geometric descriptor of many-particle systems, ${ }^{16}$ and can be calculated from the coordinates of the mass centers of the islands. In what follows, we apply this tool to the interesting case study of $\mathrm{Ge}$ dots on an oxidized $\mathrm{Si}$ surface. We prepared our samples following a procedure described in more detail

${ }^{a)}$ Electronic mail: marco.bernardi@qut.edu.au. elsewhere. ${ }^{9,17}$ Dense arrays of holes $\left(4 \times 10^{10}\right.$ holes $\left./ \mathrm{cm}^{2}\right)$ were produced by focused ion beam (FIB) milling selected areas of a Si substrate. The holes $(30 \pm 2 \mathrm{~nm}$ in diameter) are arranged in a square pattern with a pitch distance of $48 \pm 3 \mathrm{~nm}$, as measured ex situ by atomic force microscopy (AFM). The final sample topography is a mix of FIB patterned and nonpatterned flat areas. A chemical procedure ${ }^{9}$ was used to reduce $\mathrm{Ga}$ contamination from FIB processing below the surface concentration of $10^{16} \mathrm{~cm}^{-2}$. A two-step oxidation and chemical etching process developed a $5 \mathrm{~nm}$ thick surface layer of clean $\mathrm{SiO}_{2}$ before the sample was loaded into the growth chamber. A thin film of Ge $(4.8 \mathrm{ML}$ in thickness) was then deposited in UHV at room temperature and subsequently annealed for $20 \mathrm{~min}$ at $773 \mathrm{~K}$ to form $\mathrm{Ge}$ droplets onto the $\mathrm{SiO}_{2}$ surface. After the depositionannealing cycle, ex situ AFM imaging was used to check the resulting morphology, separately on patterned and nonpatterned areas. On patterned regions Ge droplets exhibit very good ordering, with a density up to $3.5 \times 10^{10} \mathrm{dots} / \mathrm{cm}^{2}$ and an average diameter of $\sim 40 \mathrm{~nm}$. Nonpatterned areas are homogeneously covered with Ge dots of average diameter $30 \mathrm{~nm}$ and seemingly no lateral order. Images of these two cases are shown in Figs. 1(a) and 1(b), respectively, and constitute the starting point of our analysis.

It is known that FIB milled holes are favored nucleation centers, ${ }^{9,18}$ and an arrangement of dots similar to the underlying holes pattern is thus expected on patterned regions upon annealing. Nevertheless, in nonpatterned regions some degree of order is expected as well, as a consequence of the nucleation mechanism itself. ${ }^{13,14}$ As a matter of fact, the capture zone $(\mathrm{CZ})$ induced by adatom-diffusion process ${ }^{19}$ determines a decrease in the probability of nucleation around each dot, impeding the formation of two very close islands. This acts as an effective repulsion between dots producing deviations from their random distribution. In this perspective, it is interesting to assess nanoislands ordering in both nanopatterned and nonpatterned regions, and we use for this purpose a software-graphic routine developed by Berrilli et al. ${ }^{20}$ to calculate the PDF. The islands mass centers are detected in the image [Fig. 1(c)] with only minor lapses, and from their coordinates the PDF is then calculated. For our computational scopes we define the PDF as ${ }^{21}$ 


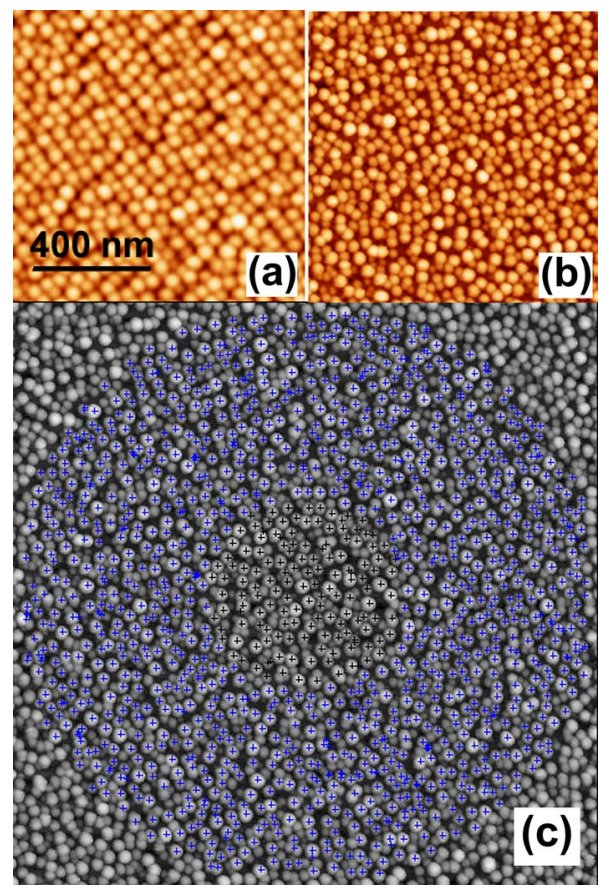

FIG. 1. (Color online) $2 \times 2 \mu \mathrm{m}^{2}$ AFM images of Ge islands on oxidized $\mathrm{Si}(100)$ surface, analysed using the PDF method. (a) FIB patterned surface, showing an arrangement of QDs resembling that of the underlying holes square pattern. (b) Nonpatterned sample, with seemingly random spatial arrangement. (c) Image (b) showing the mass center of each dot as detected in the first step of the software routine.

$$
g(r)=\frac{N(r)}{2 \pi r \Delta r \rho},
$$

where, centering on each dot, $N(r)$ is the number of dots found in the circular crown of area $2 \pi r \Delta r$ at distance $r, \Delta r$ is the bin size of the distances distribution, and $\rho$ is the mean dot density in the image. The PDF, as defined in (1), has an asymptotic value of 1 , and deviations from unity are interpreted as fluctuations from the mean density at a given distance $r$ from a dot, within a bin size $\Delta r$. Our analysis requires a relatively large statistical sample, say 1000-2000 islands, takes into account edge effects ${ }^{22}$ and is accomplished in a few seconds on a standard computer. We consider the cases of patterned and nonpatterned surface separately.

On patterned areas we observe QDs nucleation around or inside the holes upon annealing. When our software routine is run on the related image [Fig. 1(a)] the PDF function is calculated. With reference to Fig. 2, we note the occurrence of several peaks with a value well above 1 , each peak corresponding to a single or multiple (depending on the chosen bin size) shell of dots. These features show the presence of LRO. The first peak occurs at a nearest neighbor distance (NND) of $57 \pm 3 \mathrm{~nm}$, which is slightly larger than the pattern pitch, while the inflection point is located at $40 \mathrm{~nm}$, i.e., the same value as the islands average diameter (some islands touch). In an ideal square lattice of points, PDF peaks occur at distances (in units of the lattice parameter $a$ ) of $1, \sqrt{2}, 2$, $\sqrt{5}$, etc. This is experimentally found only when $\Delta / L \ll 1$, where $\Delta$ is the displacement of the nanostructure about the ideal lattice point and $L$ is the pitch distance. Due to current limits in nanostructures positioning and surface patterning, such a technologically relevant condition is hardly achieved. In our case, where $\Delta / L \approx 0.3$, we attribute deviations from the ideal case to the finite size of the dots and to the fact that

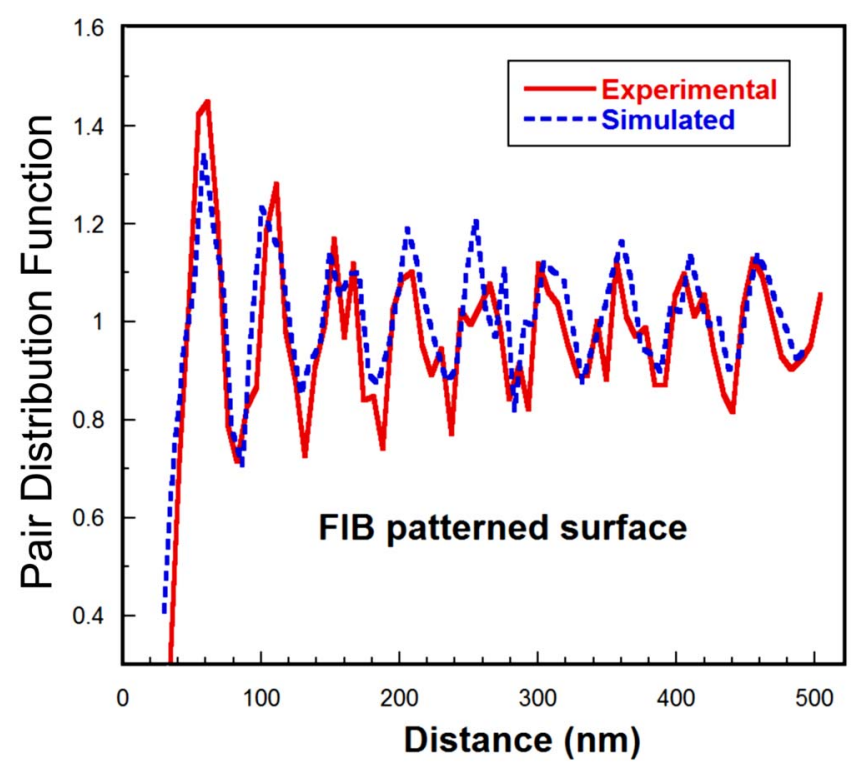

FIG. 2. (Color online) Plots of two PDFs for islands on a FIB patterned surface. The solid curve is obtained by analyzing the image reported in Fig. 1(a), and the dashed curve is the PDF of an equivalent simulated dot pattern. Both curves show evidences of LRO.

their nucleation center is located either inside or beside the hole, depending on local nanotopography. ${ }^{17,18}$ To prove this hypothesis, we model the experimental PDF using a twodimensional square lattice of points with $a=50 \mathrm{~nm}$, much like the holes pattern prepared with FIB. To account for the finite dots size and uncertainty on nucleation site, we position a single dot at random in a $30 \mathrm{~nm}$ diameter circle centered on each lattice point. The PDF extracted from this simulated pattern (Fig. 2) is strikingly similar to the one obtained from experimental data. This confirms that, when Ge nucleates in absence of strain, one dot forms at each hole of the FIB pattern, in our experimental conditions and presumably as long as the diffusion length is larger than the pitch distance. The likeness of the holes density in the FIB pattern and the QDs density $\left[1 /(50 \mathrm{~nm})^{2}=4 \times 10^{10} \mathrm{~cm}^{-2}\right.$ versus $3.5 \times 10^{10} \mathrm{~cm}^{-2}$ as found experimentally, respectively] supports further our interpretation.

A similar analysis is used for the islands in the nonpatterned areas, and the related PDFs are shown in Fig. 3. A single peak is found at the NND of $49 \pm 3 \mathrm{~nm}$ (i.e., $20 \%$ smaller than what found in the case of patterned surface) while at larger distances noise is responsible for the high frequency fluctuations of the PDF around 1. It is worth noting that, for a completely random (gaslike) distribution of pointlike QDs, $g(r) \approx 1$ at any distance $r$, meaning lack of correlation between dots. The presence of a single peak rather suggests that an interdot interaction creates a welldefined distance for the nearest neighbor (NN) shell. Since strain fields are ruled out by the amorphous $\mathrm{SiO}_{2}$ layer, it is plausible that an effective NN repulsion is solely caused by the $\mathrm{CZ}$ around each nucleated dot. An effect of self-ordering, totally hidden from visual inspection, is thus uncovered by our analysis. Moreover, differently from other studies ${ }^{13}$ we are able to exclude long-range interactions (e.g., between dipoles) relevant to LRO observing that no additional peaks occur in the PDF. Once again, we modeled the QDs pattern assuming that CZs formation is responsible for the islands arrangement. For this purpose, we altered a random distribu- 


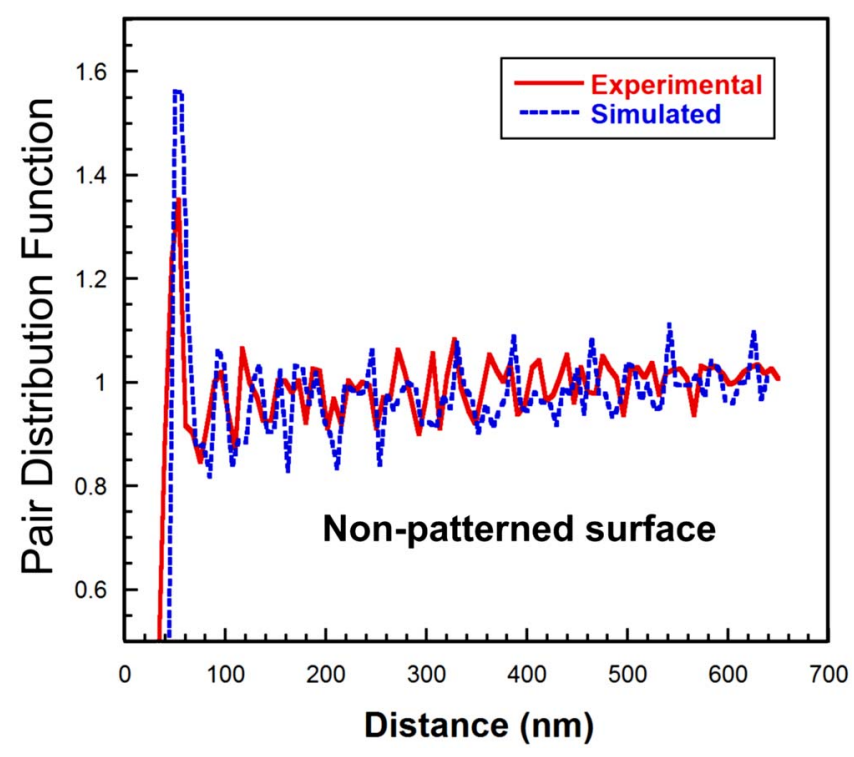

FIG. 3. (Color online) PDF of experimental (solid curve) and simulated (dashed curve) images of Ge QDs on nonpatterned Si oxide surface. The first peak at NND is a consequence of the SRO existing within the dots pattern, while the noisy signal at higher distances demonstrates that no LRO is present.

tion of islands by imposing the presence of a nucleation forbidden zone ${ }^{19}$ (NFZ) consisting of a circle, centered on each dot, whose diameter is set to $0.8 \mathrm{NND}=40 \mathrm{~nm} .{ }^{23}$ Inside the NFZ the probability of island nucleation is zero, whereas in the conventional $\mathrm{CZ}$ picture the probability of nucleation around a stable nucleus is finite, albeit reduced. Apart from minor deviations, the resulting simulated PDF resembles extremely well the experimental one (Fig. 3). It is clear that, apart from the first peak, the noise level in our data does not allow us to identify further eventual physically significant deviations from unity of the simulated PDF, and we can safely conclude that the proposed nucleation-capture mechanism is responsible for QDs self-ordering in our experimental conditions.

Remarkably, we were able to detect the significant physical mechanisms underlying self-organization of QDs in two cases of technological interest quickly and relatively effortlessly. The main advantage of our analysis is indeed the capability to assess effects of SRO in many situations of technological interest, while retaining information on LRO. In summary, we have used the PDF as a versatile tool to detect any degree of ordering of nanostructures at surfaces, hoping such a technique will assist the development of applications based on QDs and related nanostructures, where an ultimate control over self-ordering would prove crucial. Ongoing research aims at studying the dynamic PDF in systems where order among QDs varies in time in response to sudden chemical modification of the surface. ${ }^{24,25}$

The authors wish to gratefully thank Dr. Dario Del Moro for kindly sharing both his software and his expertise in the image analysis process. We also acknowledge the kind assistance of Dr. Ernesto Placidi for the AFM measurements and the insightful discussion that followed.

${ }^{1}$ J. V. Barth, G. Costantini, and K. Kern, Nature (London) 437, 671 (2005).

${ }^{2}$ R. L. Whetten, Nat. Mater. 5, 259 (2006).

${ }^{3}$ M. D. Rauscher, A. Boyne, S. A. Dregia, and S. A. Akbar, Adv. Mater. (Weinheim, Ger.) 20, 1699 (2008).

${ }^{4}$ F. M. Ross, R. M. Tromp, and M. C. Reuter, Science 286, 1931 (1999).

${ }^{5}$ Nanoparticles: From Theory to Applications, edited by G. Schmid (WileyVCH, Weinheim, 2004).

${ }^{6}$ J. De Blauwe, IEEE Trans. Nanotechnol. 1, 72 (2002).

${ }^{7}$ J. Dufourcq, S. Bodnar, G. Gay, D. Lafond, P. Mur, G. Molas, J. P. Nieto,

L. Vandroux, L. Jodin, F. Gustavo, and T. Baron, Appl. Phys. Lett. 92, 073102 (2008).

${ }^{8}$ F. Patella, A. Sgarlata, F. Arciprete, S. Nufris, P. D. Szkutnik, E. Placidi, M. Fanfoni, N. Motta, and A. Balzarotti, J. Phys.: Condens. Matter 16, S1503 (2004).

${ }^{9}$ N. Motta, P. D. Szkutnik, M. Tomellini, A. Sgarlata, M. Fanfoni, F. Patella, and A. Balzarotti, C. R. Phys. 7, 1046 (2006).

${ }^{10}$ A. Pascale, I. Berbezier, A. Ronda, and P. C. Kelires, Phys. Rev. B 77, 075311 (2008).

${ }^{11}$ P. M. Lytvyn, V. V. Strelchuk, O. F. Kolomys, I. V. Prokopenko, M. Y. Valakh, Y. I. Mazur, Z. M. Wang, G. J. Salamo, and M. Hanke, Appl. Phys. Lett. 91, 173118 (2007).

${ }^{12}$ G. Malescio and G. Pellicane, Nat. Mater. 2, 97 (2003).

${ }^{13}$ F. Ratto, T. W. Johnston, S. Heun, and F. Rosei, Surf. Sci. 602, 249 (2008).

${ }^{14}$ F. Ratto, A. Locatelli, S. Fontana, S. Kharrazi, S. Ashtaputre, S. K. Kulkarni, S. Heun, and F. Rosei, Phys. Rev. Lett. 96, 096103 (2006).

${ }^{15} \mathrm{E}$. Y. Vedmedenko, Competing Interactions and Pattern Formation in Nanoworld (Wiley-VCH, Weinheim, 2007).

${ }^{16}$ O. U. Uchea, F. H. Stillinger, and S. Torquato, Physica A 360, 21 (2006).

${ }^{17}$ P. D. Szkutnik, A. Sgarlata, N. Motta, E. Placidi, I. Berbezier, and A. Balzarotti, Surf. Sci. 601, 2778 (2007).

${ }^{18}$ A. Portavoce, R. Hull, M. C. Reuter, and F. M. Ross, Phys. Rev. B 76, 235301 (2007).

${ }^{19}$ M. Fanfoni and M. Tomellini, J. Phys.: Condens. Matter 17, R571 (2005).

${ }^{20}$ F. Berrilli, D. Del Moro, G. Consolini, E. Pietropaolo, T. L. Duvall, Jr., and A. G. Kosovichev, Sol. Phys. 221, 33 (2004).

${ }^{21}$ J. P. Hansen and I. R. McDonald, Theory of Simple Liquids, 3rd ed. (Academic, Amsterdam, 1990).

${ }^{22}$ Two concentric circles around the center of a square image are considered. The diameter of the outer circle is the image side $L$, while the inner circle diameter is $L / 3$. Only dots within the inner circle are used for the $g(r)$ calculation, while the dots in the annulus between the two circles serve as a statistical ensemble [the two circles are visible in the color version of Fig. 1(c)]. Using a maximum distance $r_{\max }=L / 3$ edge effects are correctly taken into account. This in turn reduces the sampled structures to a fraction of $\pi / 36 \approx 0.09$ of the total, making the presence of a relatively large number of islands necessary.

${ }^{23}$ Since during the annealing a maximum number of dots compatible with the presence of the $\mathrm{CZ}$ would reasonably form, the simulated pattern ought to be created with the same criterion, that is, a maximum dots density is to be used with the NFZ size as a constraint. As a bonus, this jammed packing of CZs leads to little variations in the PDF in repeated patterns generation, which is usually untrue for simulations of correlated dots patterns in which a jamming density is not attained.

${ }^{24}$ G. Capellini, M. De Seta, F. Evangelisti, V. A. Zinovyev, G. Vastola, F. Montalenti, and L. Miglio, Phys. Rev. Lett. 96, 106102 (2006).

${ }^{25}$ U. Denker, A. Rastelli, M. Stoffel, J. Tersoff, G. Katsaros, G. Costantini, K. Kern, N. Y. Jin-Phillipp, D. E. Jesson, and O. G. Schmidt, Phys. Rev. Lett. 94, 216103 (2005). 